Review

\title{
Intracranial CNS Manifestations of Myeloid Sarcoma in Patients with Acute Myeloid Leukemia: Review of the Literature and Three Case Reports from the Author's Institution
}

\author{
Gustavo M. Cervantes * and Zuzan Cayci * \\ Department of Neuroradiology, University of Minnesota, Minneapolis, MN 55455, USA; \\ E-Mails: cerva039@umn.edu (G.M.C.); cayci001@umn.edu (Z.C.); Tel.: +1-612-626-5566; \\ Fax: +1-612-626-5505
}

Academic Editors: Celalettin Ustun and Lucy A. Godley

Received: 18 December 2014 / Accepted: 5 May 2015 / Published: 21 May 2015

\begin{abstract}
Myeloid sarcoma (MS) of the central nervous system (CNS) is a rare presentation of leukemic mass infiltration outside of the bone marrow. It may involve the subperiosteum and dura mater and, on rare occasions, can also invade the brain parenchyma. The disease is most commonly seen in children or young adults; however, it has been described in multiple age groups. MS can be seen in patients with acute myeloid leukemia (AML), chronic myeloid leukemia and other myeloproliferative disorders. This entity has the potential to be underdiagnosed if the MS appearance precedes the first diagnosis of leukemia. The main reason is that their appearance on CT and MRI has a broad differential diagnosis, and proper diagnosis of MS can only be made if the imaging findings are correlated with the clinical history and laboratory findings. Herein, we describe the intracranial CNS manifestations of MS in patients with AML on CT and MRI involving the brain and/or meninges. This study is based on a systematic review of the literature. In addition, three case reports from the author's institution with AML and intracranial involvement of MS are included. Our aim is to enhance the awareness of this entity among both clinicians and radiologists.
\end{abstract}

Keywords: AML; acute myeloid leukemia; leukemia; myeloid sarcoma; extramedullary disease; chloroma; intracranial; central nervous system; brain; spine 


\section{Introduction}

Central nervous system (CNS) myeloid sarcoma (MS), a rare manifestation of acute myeloid leukemia (AML), chronic myeloid leukemia and other myeloproliferative disorders were first described by Burns in 1811 [1] by the term chloroma. MS can develop when the immature myeloblast groups form solid tumors outside the bone marrow. Intracranially, myeloid sarcomas are often continuous with the meninges or the ependyma. Nevertheless, on rare occasions, myeloid sarcoma may invade the brain parenchyma and, thus, may appear as intra-axial masses.

According to Audouin et al., [2], there are four different patterns of myeloid sarcoma development in AML patients:

(1) They may develop during the active phase of leukemia;

(2) They may develop concurrently with known chronic myeloproliferative disorders;

(3) They may manifest as a relapse after months or years after clinical remission of AML, particularly after bone marrow transplantation;

(4) They may precede the AML diagnosis and may be detected in previously healthy patients who have a normal peripheral blood cell count and who have no blast infiltration in the bone marrow (0.6\% of cases as described by Krause [3]). In this group of patients, most of the patients develop myeloid leukemia blast infiltration an average of 10.5 months following the diagnosis of a myeloid sarcoma [4]. In a large population-based cohort study from Denmark, the prevalence of myeloid sarcoma among 2261 patients with AML was 9.7\%; however, CNS involvement by myeloid sarcoma was only present in $0.4 \%$ of these patients [5].

In this review of the literature, we have identified 21 reported cases of myeloid sarcoma in patients with AML and describe the diagnostic and imaging findings pertinent to the brain parenchyma and/or meninges. In addition, three patients from our institution with AML and positive intracranial involvement were included.

\section{Literature Review}

A PubMed database search using the descriptors chloroma, myeloid sarcoma, myeloid sarcoma and brain, intracranial, central nervous system and cranial nerves retrieved 56 relevant citations from 1971 to 2014. Reviewing the literature, 21 reported cases of myeloid sarcoma with a sufficient description of the diagnosis and imaging findings related to the brain and/or meninges were identified. Leukemic conditions other than AML, such as chronic myeloid leukemia and myeloproliferative disorders, were not included. Only articles in English that were available on the PubMed central publishing platform were considered.

\section{Results}

CT and MRI were the most commonly utilized imaging modalities for assessment of CNS myeloid sarcoma. Solely MR imaging was used in 11 reported cases, and solely CT was used in four case reports. Combined MR and CT were utilized in six reported cases. Intracranial CNS imaging 
manifestations of myeloid sarcomas were reviewed in regards to their anatomic site, intra-axial versus extra-axial location, single versus multiple lesions and CT and/or MR imaging characteristics (Table 1).

A total of 21 reported patients (ten male and ten female) with AML and CNS myeloid sarcoma were included in this review. Gender was not available for one case report [6]. Nineteen patients included were adults, one a three-year-old child and another a 16-year-old adolescent. Patients' ages ranged between three and 58 years old. Mean age at the time of diagnosis of intracranial myeloid sarcoma was 35 years. Out of the 21 reported cases, 13 patients (61\%) had preexisting AML, either in remission or in acute bone marrow relapse at the time of the first neurologic symptoms. Eight patients (38\%) presented with CNS symptoms preceding their AML diagnosis.

Out of the 21 patients, a total of 24 intracranial myeloid sarcoma lesions were described. Of those, 13 lesions (54\%) were described in the intra-axial compartment of the brain and 11 lesions (45\%) in the extra-axial brain compartment. One patient had sequential lesions develop in more than one anatomic site in the brain [7]. Another patient had concurrent intra-axial lesions described in the left occipital lobe, right temporal lobe and left cerebellum [8].

Overall, the sites of intracranial myeloid sarcomas included the temporal lobe $(n=6)$, frontal lobe $(n=4)$, cerebellum $(n=4)$, parietal lobe $(n=3)$, occipital lobe $(n=3)$, cerebellopontine angle cistern $(n=1)$, corpus callosum $(n=1)$, basal ganglia $(n=1)$ and subdural space $(n=1)$. Six patients described also showed either concurrent or sequential lesions in locations outside the brain. These extra-cranial locations included the temporal bone $(n=1)$, both kidneys $(n=1)$, multiple bones $(n=1)$, nasopharynx ( $n=1)$, cervical and lumbar spine $(n=1)$, thoracic and sacral spine $(n=1)$, scalp tissues $(n=2)$ and infratemporal fossa $(n=2)$. One intracranial vascular lesion involving the superior sagittal sinus was described in one patient $(n=1)$.

Twelve out of a total of 24 lesions were assessed with CT. Among these, 11 lesions (91\%) appeared hyperdense on noncontrast CT. Out of all 11 hyperdense lesions, six lesions were intra-axial and five extra-axial. Twenty-two lesions (91\%) exhibited avid homogeneous enhancement. One lesion demonstrated inhomogeneous thick peripheral enhancement, indistinguishable from a brain abscess [9]. In another patient, a lesion described in the right temporal lobe demonstrated avid enhancement with a central core of hypoenhancement, suggestive of necrosis [8].

Seven intra-axial MS lesions were assessed with brain MRI. MS demonstrated either a hyper, iso- or hypo-intense signal on T2-weighted images. T2 hyperintensity was described in four lesions, while T2 iso- or hypo-intensity was described in three lesions. Nine extra-axial MS lesions were assessed with MRI. T2 hyperintensity was described in two extra-axial lesions; however, T2 signal findings were not described for the remaining seven extra-axial reported lesions assessed with MRI. Primary bone destruction was evident in one patient, resulting in erosion of the petrous portion of the temporal bone and subsequent intraparenchymal involvement of the right temporal lobe [10].

Vasogenic edema surrounding the enhancing leukemic masses was identifiable on both CT and MRI. This was seen in 11 lesions (45\%). Among these, six lesions were in the extra-axial and five in the intra-axial compartment of the brain. 
Table 1. Summary of clinical findings, CT and MR features of intracranial myeloid sarcomas (MS) in 21 reported cases presenting with acute myeloid leukemia.

\begin{tabular}{|c|c|c|c|c|c|c|}
\hline Authors, Year & $\begin{array}{c}\text { Age (Years), } \\
\text { Gender }\end{array}$ & AML Diagnosis & $\begin{array}{l}\text { Single vs. } \\
\text { Multiple }\end{array}$ & CNS Anatomic Location (s) & $\begin{array}{c}\text { Intra- or } \\
\text { Extra-Axial } \\
\end{array}$ & CT and/or MR Characteristics \\
\hline Yang et al., 2014 [11] & 27, male & preceding AML diagnosis & single & occipital lobe (1), spine (2) & extra-axial & $\begin{array}{c}\text { meningioma-like, vasogenic } \\
\text { edema }\end{array}$ \\
\hline Cho et al., 2013 [12] & 27, female & AML relapse, 10 months in CR & single & right cerebellopontine angle & extra-axial & $\begin{array}{c}\text { meningioma-like, vasogenic } \\
\text { edema }\end{array}$ \\
\hline Murakami et al., 2011 [10] & 52, male & AML relapse, 5 years in CR & multiple & $\begin{array}{l}\text { right temporal bone and right } \\
\text { temporal lobe }\end{array}$ & extra-axial & $\begin{array}{c}\text { meningioma-like, vasogenic } \\
\text { edema }\end{array}$ \\
\hline Akhaddar et al., 2011 [6] & $27, \mathrm{n} / \mathrm{a}$ & preceding AML diagnosis & single & right frontal lobe & intra-axial & $\begin{array}{c}\text { intraparenchymal, vasogenic } \\
\text { edema }\end{array}$ \\
\hline Eom et al., 2011 [7] & 49 , male & AML relapse, 2 years in CR & multiple & $\begin{array}{l}\text { left cerebellum, right frontal lobe } \\
\text { and cervical and lumbar spine }\end{array}$ & intra-axial & intraparenchymal \\
\hline Cho et al., 2010 [13] & 44, male & AML relapse, 2 years in CR & single & corpus callosum & intra-axial & meningioma-like \\
\hline Grier et al., 2008 [14] & 41 , female & preceding AML diagnosis & multiple & $\begin{array}{l}\text { left temporal lobe, } \\
\text { infratemporal extension }\end{array}$ & extra-axial & meningioma-like \\
\hline Widhalm et al., 2006 [15] & 35 , female & preceding AML diagnosis & multiple & $\begin{array}{l}\text { right parietal lobe and } \\
\text { subgaleal (1), spine (2) }\end{array}$ & extra-axial & meningioma-like \\
\hline Smidt et al., 2005 [16] & 45 , male & AML relapse, 16 years in CR & single & $\begin{array}{l}\text { left cerebral hemisphere } \\
\text { subdural location }\end{array}$ & extra-axial & $\begin{array}{c}\text { hemispheric subdural mass, } \mathrm{C}+\text {, } \\
\text { mass effect }\end{array}$ \\
\hline $\begin{array}{c}\text { Best-Aguilera et al., } 2005 \\
\text { [17] }\end{array}$ & 37, male & preceding AML diagnosis & multiple & $\begin{array}{l}\text { right occipital lobe, } \\
\text { mediastinum, retroperitoneum, } \\
\text { liver, rectum }\end{array}$ & extra-axial & $\begin{array}{c}\text { meningioma-like, vasogenic } \\
\text { edema }\end{array}$ \\
\hline Nishimura et al., 2004 [18] & 30 , female & AML relapse, 16 months in CR & single & $\begin{array}{l}\text { right frontal lobe, subgaleal and } \\
\text { superior sag. sinus extension }\end{array}$ & extra-axial & $\begin{array}{c}\text { meningioma-like, vasogenic } \\
\text { edema }\end{array}$ \\
\hline
\end{tabular}


Table 1. Cont.

\begin{tabular}{|c|c|c|c|c|c|c|}
\hline Authors, Year & $\begin{array}{c}\text { Age (Years), } \\
\text { Gender }\end{array}$ & AML Diagnosis & $\begin{array}{l}\text { Single vs. } \\
\text { Multiple }\end{array}$ & CNS Anatomic Location (s) & $\begin{array}{c}\text { Intra- or } \\
\text { Extra- } \\
\text { Axial } \\
\end{array}$ & CT and/or MR Characteristics \\
\hline Suzer et al., 2004 [19] & 58 , female & $\begin{array}{l}\text { AML relapse, 6-12 months in } \\
\text { CR }\end{array}$ & single & left cerebellar hemisphere & intra-axial & Intraparenchymal \\
\hline Park et al., 2003 [20] & 3, female & preceding AML diagnosis & multiple & $\begin{array}{l}\text { right temporal lobe, } \\
\text { infratemporal extension, } \\
\text { kidneys, bones }\end{array}$ & extra-axial & meningioma-like \\
\hline Nikolic et al., 2003 [21] & 45 , male & $\begin{array}{l}\text { AML relapse, indeterminate } \\
\text { CR }\end{array}$ & single & left frontal lobe & extra-axial & $\begin{array}{c}\text { meningioma-like, vasogenic } \\
\text { edema }\end{array}$ \\
\hline Guermazi et al., 2002 [22] & 28, female & AML relapse, 8 months in CR & single & left parietal lobe & intra-axial & $\begin{array}{c}\text { intraparenchymal, vasogenic } \\
\text { edema }\end{array}$ \\
\hline Guermazi et al., 2002 [22] & 58, female & preceding AML diagnosis & single & right basal ganglia & intra-axial & $\begin{array}{c}\text { intraparenchymal, vasogenic } \\
\text { edema }\end{array}$ \\
\hline Ooi et al., 2001 [9] & 32, male & AML relapse, 18 months in CR & multiple & $\begin{array}{l}\text { right temporal lobe, } \\
\text { nasopharynx }\end{array}$ & intra-axial & $\begin{array}{l}\text { intraparenchymal, mimicking } \\
\text { abscess }\end{array}$ \\
\hline Yamamoto et al., 1999 [23] & 38, male & AML relapse, 2 years in CR & single & left temporal lobe & intra-axial & $\begin{array}{c}\text { intraparenchymal, vasogenic } \\
\text { edema }\end{array}$ \\
\hline Parker et al., 2005 [24] & 29, female & AML relapse, 6 months in CR & single & cerebellar vermis & intra-axial & intraparenchymal \\
\hline Yoon et al., 1987 [25] & 16 , female & preceding AML diagnosis & single & left parietal lobe & extra-axial & meningioma-like \\
\hline Barnett, Zussman 1986 [8] * & 34 , male & AML relapse, 13 months in CR & multiple & $\begin{array}{l}\text { left occipital and left } \\
\text { cerebellum }\end{array}$ & $\begin{array}{l}\text { intra-axial } \\
\text { (2) }\end{array}$ & intraparenchymal lesions \\
\hline Barnett, Zussman 1986 [8] * & 34 , male & AML relapse, 13 months in CR & multiple & right temporal lobe & $\begin{array}{l}\text { intra-axial } \\
(1) \\
\end{array}$ & $\begin{array}{c}\text { intraparenchymal, hypodense } \\
\text { lesion, core of necrosis }\end{array}$ \\
\hline
\end{tabular}

Asterisks $\left(^{*}\right)$ represent a case report from the literature with three concurrent lesions described, which were separated into two paragraphs, because of the peculiar imaging findings of the right temporal lobe lesion; numbers in parentheses represent numerical values of the lesions described; n/a, not available. 


\section{Authors' Institution Case Reports}

CT and MR imaging characteristics of three patients from our institution presenting with acute myeloid leukemia and intracranial CNS manifestations of myeloid sarcoma are described. Case 3 was previously reported in the literature by one of the authors [26].

Case 1: A 69-year-old female patient with a recent history of worsening headaches and flu-like symptoms diagnosed with AML and more than 90\% myeloid cells in the blood count during her first admission (Figure 1).

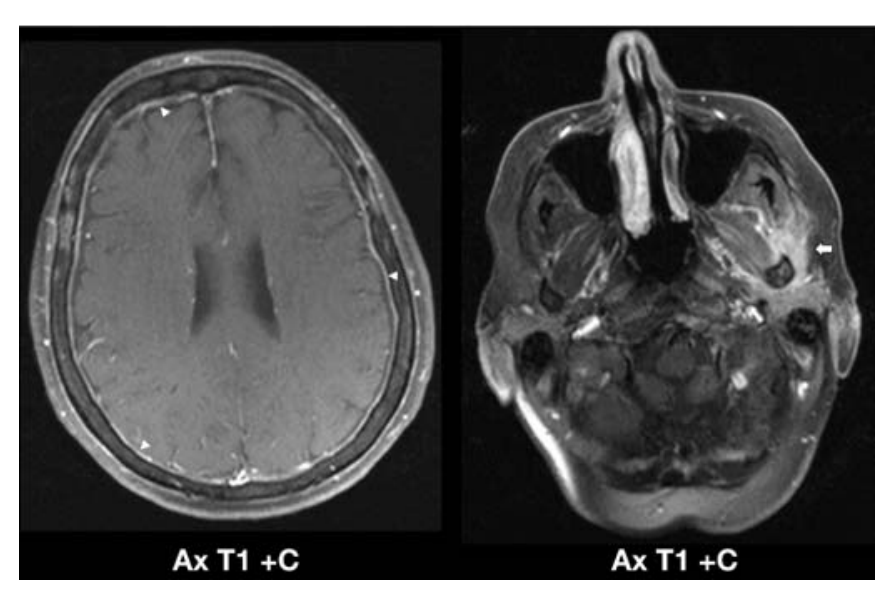

Figure 1. Meningeal infiltration related to AML in a previously healthy 69-year-old woman. Axial post-contrast T1-weighted images with fat saturation (left image) demonstrate marked pachymeningeal enhancement (arrowheads), most consistent with leukemic infiltration of the brain meninges. Note the marked ill-defined enhancement of the left infratemporal fossa (right image) surrounding the left masticator and deep parotid spaces (arrow), corresponding to extra-cranial leukemic myeloid sarcoma of the soft tissues of the left neck. Complete resolution of the leukemic infiltration within the deep left neck was observed after successful AML induction chemotherapy on follow-up imaging (not shown). Ax, axial; C+, post-contrast.

Case 2: A 56-year-old female patient with acute myelomonocytic leukemia, French-American-British (FAB) classification M4, developed a first relapse of AML with 26\% blasts in the blood count during admission. She underwent double umbilical cord blood stem cell transplantation during the remission phase after being successfully treated with induction chemotherapy for her first relapse. On Day 39 of the post-transplant period, she presented with persistent headaches, muscle weakness, falls at home and the onset of leukemia cutis with new skin lesions (Figure 2). 


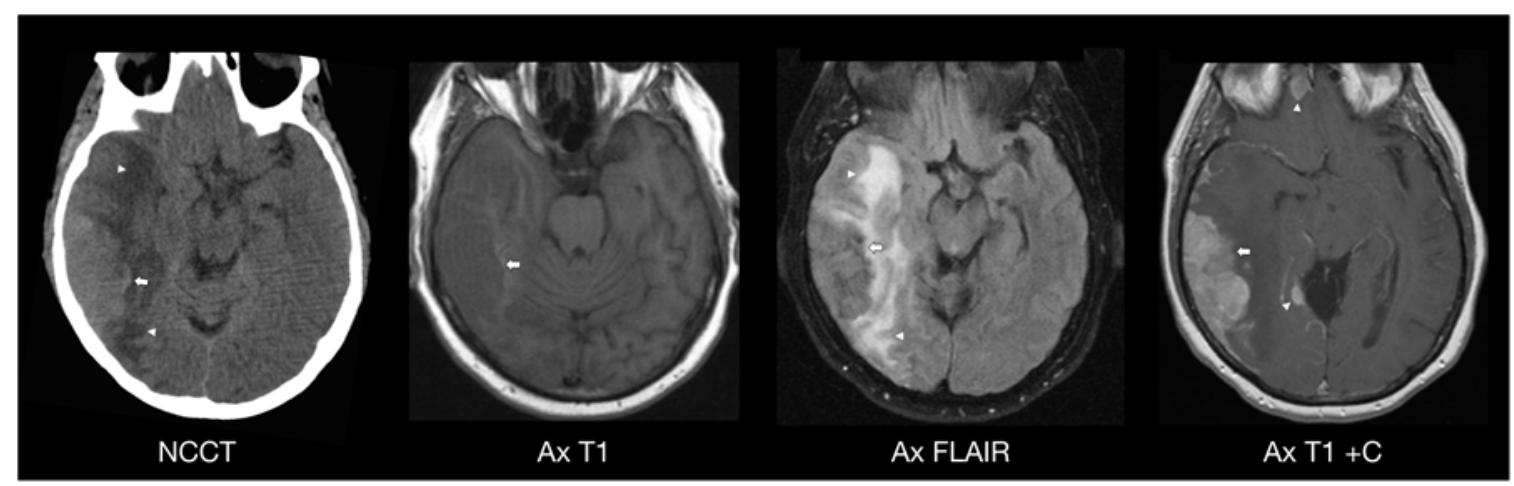

Figure 2. AML relapse in the form of a myeloid sarcoma mass lesion involving the right temporal lobe of a 56-year-old woman 39 days after umbilical cord blood stem cell transplantation. Noncontrast CT images demonstrate a large hyperdense dural-based mass lesion involving the right temporal lobe (NCCT, arrow) with surrounding edema (NCCT, arrowheads), mass effect and midline shift. On MRI, a large ill-defined avidly enhancing dural-based mass lesion involving the posterior aspect of the right temporal lobe (Ax T1 $\mathrm{C}+$, arrow) with surrounding vasogenic edema (Ax FLAIR, arrowheads) was noted. This infiltrating mass revealed hypointense signal intensity on $\mathrm{T} 1$ ( $\mathrm{Ax} \mathrm{T} 1$, arrow) and T2-weighted images relative to the adjacent gray-matter. Diffusion weighted images (not shown) demonstrated restricted diffusion in the posterolateral aspect of the right temporal lobe mass consistent with increased cellularity. Additional pachymeningeal foci of nodular enhancement were noted within the gyrus rectus of the right inferior frontal lobe and within the medial aspect of the right inferior temporal lobe (Ax T1 C+, arrowheads). Diffuse right hemispheric pachymeningeal enhancement was also noted (Ax T1 C+, partially shown). Ax, axial; Sag, sagittal; FS, fat saturation; $\mathrm{C}+$, post-contrast; DWI, diffusion-weighted images; ADC, apparent diffusion coefficient.

Case 3: A 53-year-old man with a three-month history of polydipsia and polyuria and indication of myelodysplastic syndrome (MDS) presented with refractory anemia and 16\% blast cells in the bone marrow biopsy suggesting a diagnosis of refractory anemia with excess of blasts (RAEB). Diabetes insipidus was confirmed by inappropriately low urine osmolality and low antidiuretic hormone (ADH) levels. AML induction chemotherapy (seven days of cytarabine and three days of idarubicin) along with intrathecal methotrexate after cerebrospinal fluid (CSF) analysis was initiated (Figure 3). 


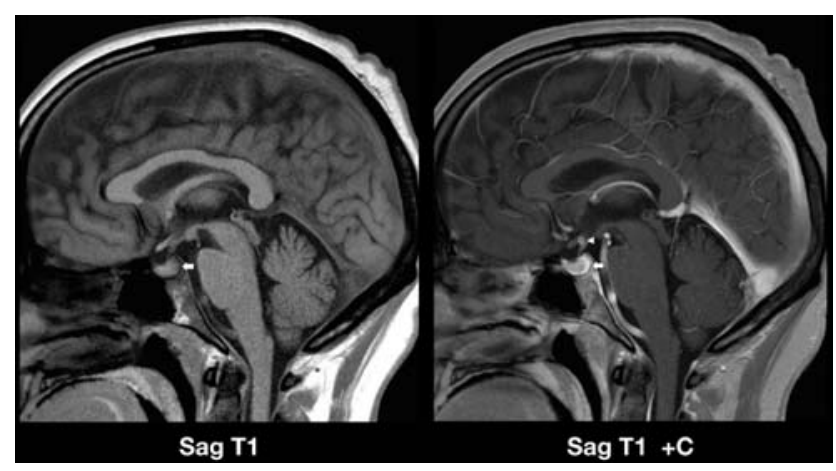

Figure 3. Leukemic infiltration of the neurohypophysis and pituitary stalk in a patient with myelodysplastic syndrome presenting with diabetes insipidus. The sagittal T1-weighted image without contrast administration (left image) demonstrates the absence of the neurohypophysis (arrow), confirming the diagnosis of diabetes insipidus. Normally, the neurohypophysis is identified as a bright signal spot on T1-weighted images in the posterior pituitary gland. The sagittal T1-weighted image after contrast administration (right image) reveals a 2-mm enhancing nodular lesion in the superior aspect of the pituitary stalk (arrowhead) and curvilinear enhancement along the posterior aspect of the pituitary gland in the expected region of the neurohypophysis (arrow). Sag, sagittal; $\mathrm{C}+$, post-contrast.

\section{Discussion}

CNS leukemic infiltration may present in one or more of the following intracranial forms: (1) meningeal disease, as "carcinomatous meningitis"; (2) intravascular tumor aggregates throughout the brain, as "carcinomatous encephalitis"; or (3) focal tumor masses, as "myeloid sarcomas”. When AML manifests as a solid tumor outside the bone marrow, i.e., myeloid sarcoma, the imaging features can be mistaken for some conditions other than the myeloid sarcoma. Myeloid sarcoma seen in leukemic patients may involve any part of the body. The most common sites for myeloid sarcoma deposits are the bones, soft tissues, lymph nodes and the skin. Rarely, they may manifest as single or multiple intracranial lesions [4], most commonly seen within the calvaria and orbits [27]. In our review, out of 24 intracranial MS lesions reviewed from the literature, 13 lesions (48\%) have presented as focal tumor masses within the intraparenchymal compartment of the brain.

Myeloid sarcoma can occur at variable ages (1-81 years old), often after the diagnosis of AML. However, in approximately 25\% of the patients, MS appears before the initial diagnosis of AML by months or years [28]. According to Neiman et al., of a total of 61 biopsied proven myeloid sarcomas, twenty-two lesions were seen in 15 patients with no known history of acute myeloid leukemia [4]. Most of these patients developed acute leukemia on an average of 10.5 months after the biopsy. In this review, eight out of 21 reported cases (38\%) had intracranial CNS imaging manifestations of myeloid sarcoma preceding the diagnosis of AML.

Radiologically, intracranial MS in patients with acute myeloid leukemia most commonly presents as an extra-axial hyperdense mass on noncontrast CT scan. In this review of the literature, all 24 intracranial lesions, except for one, appear as hyperdense masses on the noncontrast CT studies. The differential diagnosis of hyperdense intracranial masses is broad and includes meningioma, B-cell lymphoma and intracranial metastasis. Interestingly, myeloid sarcomas can express B-cell antigens (e.g., 
CD19, CD79a) and, thus, potentially lead to a histologic misdiagnosis of CNS lymphoma, if no immunohistochemistry or flow cytometry analysis is available [14]. Other less common diagnostic considerations considered are metastatic neuroblastoma (in the pediatric age group), Ewing's sarcoma, hemangiopericytoma and subdural hematoma. A second pattern of presentation is patchy and/or leptomeningeal enhancement seen in patients with acute myeloid leukemia. In this situation, the differential diagnosis to consider is: primary meningeal tumor infiltration by leukemia, secondary leptomeningeal carcinomatosis, viral or bacterial meningitis, neurosarcoidosis, dural sinus thrombosis, intracranial hypotension or postoperative changes.

Vasogenic edema was present on either CT or MR in 11 lesions (45\%) out of the 24 intracranial myeloid sarcomas that were included in this review. Further characterization of other CT and/or MR imaging features should be used to distinguish the intracranial MS lesions from other commonly-found intracranial lesions, such as meningioma, lymphoma or brain metastasis, which also commonly show vasogenic edema and can potentially occur concurrently with myeloid sarcoma in patients with acute myeloid leukemia.

Leukemic cell infiltrates are capable of migration from the bone marrow of the periosteum and dura mater into the underlying brain parenchyma once there is disruption of the pial-glial barrier. Despite the close involvement of the extra-cranial and intra-cranial tissues, destructive bony changes are not commonly seen with myeloid sarcomas. Only one patient showed apparent bone destruction of the temporal bone and concomitant involvement of the adjacent right temporal lobe parenchyma [10].

One of the limitations of this study is the small sample of the reported cases available in the literature, since we exclusively included AML patients with intracranial MS lesions. Other forms of acute or chronic leukemias and myeloproliferative disorders could also present with intracranial MS lesions and were not included in this study. Potential selection bias of the study population is another limitation, since the majority of patients included were young adults. Since only one pediatric patient with AML and intracranial CNS myeloid sarcoma was present in our systematic review, our findings cannot be generalized to all age groups.

\section{Conclusions}

Myeloid sarcoma of the intracranial CNS is a rare presentation of AML. This can be detected before the diagnosis of leukemia or any time throughout the course of the disease during routine neuroimaging studies. Proper CT and MR imaging interpretation in conjunction with relevant clinical information, including the age at the time of diagnosis, symptomatology, such as the presence of headaches, seizures, focal neurological deficits and/or cranial nerve palsy (-ies), a history of hematologic malignancies, prior bone marrow or solid organ transplantation status and immune system status, can help to narrow the clinical diagnosis of intracranial MS. Finally, prompt clinical and laboratory evaluation for acute myeloid leukemia relapse is necessary in patients with a known history of leukemia presenting with meningeal and/or parenchymal lesions.

\section{Author Contributions}

The authors contributed equally to the manuscript. 


\section{Conflicts of Interest}

The authors declare no conflict of interest.

\section{References}

1. Burns, A. Observations of surgical anatomy. In Head and Neck; Royce: London, UK, 1811; p. 364.

2. Audouin, J.; Comperat, E.; le Tourneau, A.; Camilleri-Broët, S.; Adida, C.; Molina, T.; Diebold, J. Myeloid sarcoma: Clinical and morphologic criteria useful for diagnosis. Int. J. Surg. Pathol. 2003, 11, 271-282.

3. Krause, J.R. Myeloid sarcoma preceding acute leukemia: A report of six cases. Cancer 1979, 44, 1017-1021.

4. Neiman, R.S.; Barcos, M.; Berard, C.; Bonner, H.; Mann, R.; Rydell, R.E.; Bennett, J.M. Myeloid sarcoma: A clinicopathologic study of 61 biopsied cases. Cancer 1981, 48, 1426-1437.

5. Østgaard, L.S.G.; Sengeløv, H.; Holm, M.S.; Johnsen, H.E.; Severinsen, M.T.; Kjeldsen, E.; Bergmann, O.J.; Friis, L.S.; Jensen, M.K.; Nielsen, O.J.; et al. Extramedullary Leukemia and Myeloid Sarcoma in AML: New Clues to an Old Issue. Results of a Retrospective Population-Based Registry-Study of 2261 patients. In Annals of 53rd American Society of Hematology Annual Meeting and Exposition. San Diego, CA, USA, 10-13 December 2011.

6. Akhaddar, A.; Zyani, M.; Mikdame, M.; Boucetta, M. Acute myeloid leukemia with brain involvement (chloroma). Intern. Med. 2011, 50, 535-536.

7. Eom, K.S.; Kim, T.Y. Intraparenchymal myeloid sarcoma and subsequent spinal myeloid sarcoma for acute myeloblastic leukemia. J. Korean Neurosurg. Soc. 2011, 49, 171-174.

8. Barnett, M.J.; Zussman, W.V. Granulocytic sarcoma of the brain: a case report and review of the literature. Radiology 1986, 160, 223-225.

9. Ooi, G.C.; Chim, C.S.; Khong, P.L.; Au, W.Y.; Lie, A.K.; Tsang, K.W.; Kwong, Y.L. Radiologic manifestations of myeloid sarcoma in adult leukemia. AJR Am. J. Roentgenol. 2001, 176, 1427-1431.

10. Murakami, M.; Uno, T.; Nakaguchi, H.; Yamada, S.M.; Hoya, K.; Yamazaki, K.; Ishida, Y.; Matsuno, A. Isolated recurrence of intracranial and temporal bone myeloid sarcoma—Case report. Neurol. Med. Chir. Tokyo 2011, 51, 850-854.

11. Yang, C.; Liu, Y.; Li, G.; Bai, J.; Qian, J.; Xu, Y. Multifocal myeloid sarcoma in the central nervous system without leukemia. Clin. Neurol. Neurosurg. 2014, 120, 99-102.

12. Cho, S.F.; Liu, T.C.; Chang, C.S. Isolated central nervous system relapse presenting as myeloid sarcoma of acute myeloid leukemia after allogeneic peripheral blood stem cell transplantation. Ann. Hematol. 2013, 92, 133-135.

13. Cho, W.H.; Choi, Y.J.; Choi, B.K.; Cha, S.H. Isolated recurrence of intracranial granulocytic sarcoma mimicking a falx meningioma in acute myeloblastic leukemia. J. Korean Neurosurg. Soc. 2010, 47, 385-388.

14. Grier, D.D.; Al-Quran, S.Z.; Gray, B.; Li, Y.; Braylan, R. Intracranial myeloid sarcoma. Br. J. Haematol. 2008, 142, 681. 
15. Widhalm, G.; Dietrich, W.; Müllauer, L.; Streubel, B.; Rabitsch, W.; Kotter, M.R.; Knosp, E.; Roessler, K. Myeloid sarcoma with multiple lesions of the central nervous system in a patient without leukemia. Case report. J. Neurosurg. 2006, 105, 916-919.

16. Smidt, M.H.; de Bruin, H.G.; van’t Veer, M.B.; van den Bent, M.J. Intracranial granulocytic sarcoma (chloroma) may mimic a subdural hematoma. J. Neurol. 2005, 252, 498-499.

17. Best-Aguilera, C.R.; Vazquez-Del Mercado, M.; Muñoz-Valle, J.F.; Herrera-Zarate, L.; Bonilla, G.M.; Navarro-Hernandez, R.E.; Martin-Marquez, B.T.; Oregon-Romero, E.; Ruiz-Quezada, S.; Lomeli-Guerrero, A. Massive myeloid sarcoma affecting the central nervous system, mediastinum, retroperitoneum, liver, and rectum associated with acute myeloblastic leukaemia: A case report. J. Clin. Pathol. 2005, 58, 325-357.

18. Nishimura, S.; Kyuma, Y.; Kamijo, A.; Maruta, A. Isolated recurrence of granulocytic sarcoma manifesting as extra- and intracranial masses_Case report. Neurol. Med. Chir. Tokyo 2004, 44, 311-316.

19. Suzer, T.; Colakoglu, N.; Cirak, B.; Keskin, A.; Coskun, E.; Tahta, K. Intracerebellar granulocytic sarcoma complicating acute myelogenous leukemia: A case report and review of the literature. J. Clin. Neurosci. 2004, 11, 914-917.

20. Park, H.J.; Jeong, D.H.; Song, H.G.; Lee, G.K.; Han, G.S.; Cha, S.H.; Ha, T.S. Myeloid sarcoma of both kidneys, the brain, and multiple bones in a nonleukemic child. Yonsei Med. J. 2003, 44, 740-743.

21. Nikolic, B.; Feigenbaum, F.; Abbara, S.; Martuza, R.L.; Schellinger, D. CT changes of an intracranial granulocytic sarcoma on short-term follow-up. AJR Am. J. Roentgenol. 2003, 180, 78-80.

22. Guermazi, A.; Feger, C.; Rousselot, P.; Merad, M.; Benchaib, N.; Bourrier, P.; Mariette, X.; Frija, J.; de Kerviler, E. Granulocytic sarcoma (chloroma): Imaging findings in adults and children. AJR Am. J. Roentgenol. 2002, 178, 319-325.

23. Yamamoto, K.; Hamaguchi, H.; Nagata, K.; Hara, M.; Tone, O.; Tomita, H.; Ito, U. Isolated recurrence of granulocytic sarcoma of the brain: Successful treatment with surgical resection, intrathecal injection, irradiation and prophylactic systemic chemotherapy. Jpn. J. Clin. Oncol. 1999, 29, 214-218.

24. Parker, K.; Hardjasudarma, M.; McClellan, R.L.; Fowler, M.R.; Milner, J.W. MR features of an intracerebellar chloroma. AJNR Am. J. Neuroradiol. 1996, 17, 1592-1594.

25. Yoon, D.H.; Cho, K.J.; Suh, Y.L.; Kim, C.W.; Chi, J.G.; Han, D.H.; Bang, Y.J.; Kim, B.K.; Kim, N.K.; Cho, H.I. Intracranial granulocytic sarcoma (chloroma) in a nonleukemic patient. J. Korean. Med. Sci. 1987, 2, 173-178.

26. Chuang, C.; Parnerkar, V.; Radulescu, A.; Hunt, M.A.; Cayci, Z.; Ustun, C. Diabetes insipidus in myelodysplastic syndrome: What we learnt from a case regarding its diagnosis, pathophysiology and management. Leuk. Lymphoma 2014, 20, 1-3.

27. Osborn, A.G. Lymphomas, hematopoietic and histiocytic tumors. In Osborn's Brain: Imaging, Pathology and Anatomy; AMIRSYS Publishing: Salt Lake City, UT, USA, 2013; pp. 668-671.

28. Avni, B.; Koren-Michowitz, M. Myeloid sarcoma: Current approach and therapeutic options. Adv. Hematol. 2011, 2, 309-316.

(C) 2015 by the authors; licensee MDPI, Basel, Switzerland. This article is an open access article distributed under the terms and conditions of the Creative Commons Attribution license (http://creativecommons.org/licenses/by/4.0/). 